\title{
Functional epoxy composites for high voltage insulation involving c-BN and reactive POSS as compatibilizer
}

\author{
Thomas Heid $^{1,2}$, Michel F Fréchette ${ }^{2}$, Eric David ${ }^{1}$ \\ ${ }^{1}$ École de technologie supérieure (ETS), Montréal, QC, Canada \\ ${ }^{2}$ Institut de recherche d'Hydro-Québec (IREQ), Varennes, QC, Canada
}

\begin{abstract}
Epoxy resins modified with only Polyhedral Oligomeric Silsesquioxanes (POSS) or cubic boron nitride (c-BN), as well as multiphase composites containing both, POSS and c-BN, were fabricated and investigated. Two different types of reactive liquid POSS with molecule sizes of approximately $2 \mathrm{~nm}$, viz. Triglycidylisobutyl-POSS (TGIBPOSS) and Glycidyl-POSS were added in contents of $1 \mathrm{wt} \%$ to form single-phase and multiphase composites. Submicrometric c-BN, in contents of $5 \mathrm{wt} \%$, was used for the respective single-phase composite as well as in the multiphase samples to create multifunctional nanostructured composites. All specimens were characterized by Broadband Dielectric Spectroscopy (BDS), dielectric breakdown experiments, Differential Scanning Calorimetry (DSC) and thermal conductivity measurements. The single-phase POSS composites have seen up to $15 \%$ improved dielectric breakdown strengths and enhanced thermal conductivities by up to $20 \%$. The single-phase c-BN composite revealed the most distinct improvement in thermal conductivity of more than $25 \%$, along with a reduction in dielectric breakdown strength of approximately 18\%. Additionally, the dielectric and thermal properties of the multiphase composites containing $1 \mathrm{wt} \%$ POSS and $5 \mathrm{wt} \% \mathrm{c}-\mathrm{BN}$, were found to be solely dictated by the submicrometric c-BN particles. SEM observations revealed a significantly improved dispersion of the c-BN particles in the presence of POSS for the multiphase composites, suggesting that POSS acts as a compatibilizer between organic epoxy matrix and inorganic filler particles.
\end{abstract}

\section{Keywords}

Compatibilizer, dielectric spectroscopy, dielectric breakdown strength, epoxy composites, polymer nanodielectric, POSS, silica, cubic boron nitride, thermal conductivity

\section{Introduction}

Insulating systems are a crucial component of any high voltage (HV) apparatus used in power generation, energy transformation or for energy transportation. However, one of the weaknesses of the organic materials used for high voltage insulation, such as epoxy resins, is that they are subject to electrical degradation [1]. Even more, the polymer itself usually features a low thermal conductivity [2-6], which is a major limiting factor for materials that are expected to undergo high electrothermal stresses.

For the last decade, there has been a deep interest in nano-composite research in the scientific community in numerous fields [7, 8], and more recently also in terms of dielectric materials [9-12]. This novel class of dielectric materials is commonly called polymer nanodielectrics. The presence of inorganic nanometric particles in the organic polymer matrix can render the resulting composites more resilient to electro-thermal stresses and improve parameters such as thermal conductivity or dielectric breakdown strength [13].

Interesting filler materials, especially in the case of epoxy resins, are Polyhedral Oligomeric Silsesquioxanes (POSS). Unlike other common inorganic nanofillers, POSS is a hybrid material that features an inorganic silica-like core and organic side-groups. When these organic side-groups are of reactive nature and compatible with the epoxy system at hand, as is the case for the POSS additives further used in this study, the POSS molecules can covalently bond with the epoxy matrix. Thus, this leads to an intimate interaction between the POSS molecules (approx. $2 \mathrm{~nm}$ in size) and the polymer, resulting in a re-structuration of the polymer network on a nanometric scale. As a result, epoxy/POSS composites have revealed notable improvements in both, dielectric and thermal performances, as published in recent studies [12, 14-16].

In an effort to further enhance the thermal conductivity of polymers, Boron Nitride (BN), a highly thermally conductive ceramic, has presented itself as a promising additive $[3,17,18]$. $\mathrm{BN}$ is a chemically

The final publication is available at Springer via http://dx.doi.org/10.1007/s10853-015-9095-9 


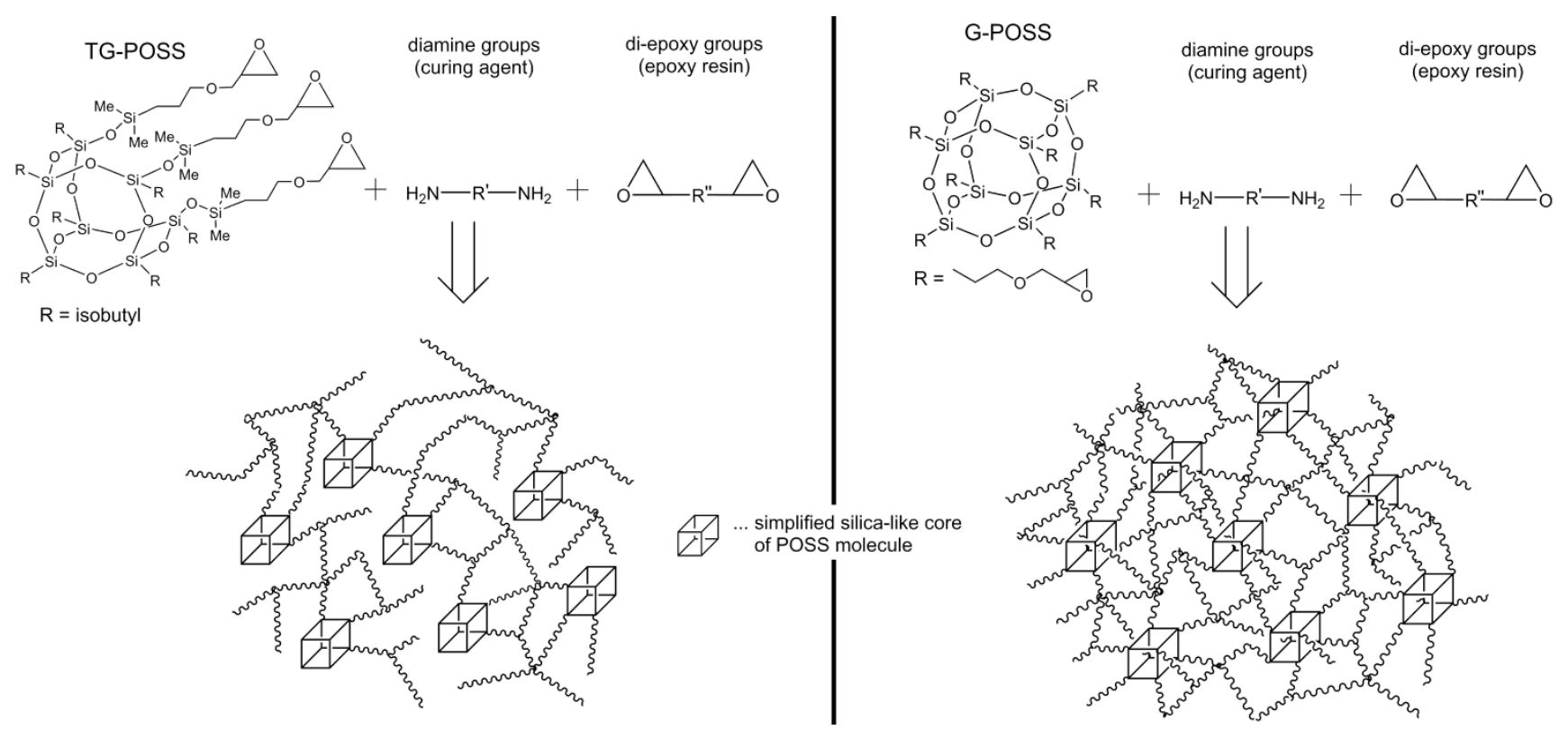

Fig. 1 Proposed reaction of the two different POSS types with curing agent and epoxy resin, TG-POSS (left) with the three epoxy groups and the G-POSS (right) with the cage structure represented for the case of $\mathrm{R}=8$ epoxy groups. The formation of covalent bonds between POSS and epoxy matrix results in nano-structured epoxy/POSS networks.

synthesized material, which has been synthesized in different crystalline phases, of which hexagonal BN (h$\mathrm{BN})$ and cubic BN (c-BN) are commonly used. Cubic BN does not occur in nature [19, 20]. Recent studies have led to the conclusion that c-BN composites have seen higher improvements of thermal conductivities compared to their h-BN counterparts [6, 21], which can be attributed to the significantly higher bulk thermal conductivity of c-BN along with its isotropic properties.

The inorganic nature of such filler particles and their tendency to agglomerate, however, can pose difficulties in terms of an intimate incorporation into an organic matrix, and for their dispersion within the polymer, when surface functionalization is not applied [22, 23].

What would happen now, if the inorganic c-BN were to be incorporated in a polymeric matrix, where POSS molecules have re-organized the polymeric web due to their reactive nature, and hence, by covalent bonding with the epoxy molecules? Could the hybrid inorganic/organic POSS, incorporated in the epoxy network, affect the interaction between c-BN and the matrix?

Therefore, to answer these questions, the scope of this work was to investigate the interaction between hybrid inorganic/organic POSS and inorganic submicrometric c-BN, when both filler types are incorporated in an epoxy composite. The expected change in morphology as a result of the covalent bonding of POSS with the epoxy molecules is assumed to affect the interaction of
c-BN particles with the epoxy/POSS network, possibly yielding a synergetic effect, where the hybrid POSS might act as a functionalizing agent, and hence, improve the integration of c-BN in the epoxy/POSS matrix.

\section{Materials and Sample Preparation}

\subsection{Materials}

DER332 epoxy resin (DOW Chemical Company) and Jeffamine D230 curing agent (Huntsman Corp.) were used as the base polymer for sample fabrication. Three distinct additives, namely a submicrometric cubic boron nitride (c-BN) with average particle sizes of $500 \mathrm{~nm}$ (from Diamond Innovations Inc.) and two different liquid reactive POSS types (from Hybrid Plastics Inc.), a Triglycidylisobutyl-POSS (TG-POSS) and a GlycidylPOSS (G-POSS), were used to fabricate composites. The TG-POSS has three mono-directionally oriented epoxy groups attached to the cage-like silica core (Fig. 1 on the left), whereas the G-POSS features 8 to 12 epoxy groups attached to the silica core (Fig. 1 on the right). Both POSS types can form covalent bonds with the epoxy system used during the curing reaction, and hence, modify the structure of the epoxy network on a nanometric level, as proposed in Fig. 1. Due to the significantly higher amount of epoxy groups featured by G-POSS, the incorporation of these molecules into the epoxy resin will lead to a higher cross-linking density of the resulting epoxy/POSS network compared to the case 
when TG-POSS is used.

The viscosity of both POSS types was comparable with that of the base epoxy, which facilitated the dispersion of POSS in the epoxy.

\subsection{Compounding}

In this study several types of composites were produced in addition to the neat epoxy (NE) reference sample: First, three composites featuring only a single additive, meaning either one of the two POSS types with a content of $1 \mathrm{wt} \%$, named G-POSS and TG-POSS respectively, or containing $5 \mathrm{wt} \%$ of the submicrometric cubic boron nitride, named cBN, were produced. The second type of composites were multiphase composites (MP), where either type of POSS was incorporated along with $5 \mathrm{wt} \%$ of c-BN. Hence, one composite with $1 \mathrm{wt} \%$ of G-POSS plus $5 \mathrm{wt} \% \mathrm{c}-\mathrm{BN}$ (MP/G-POSS) and one composite with $1 \mathrm{wt} \%$ of TG-POSS plus $5 \mathrm{wt} \%$ c-BN (MP/TG-POSS) were obtained. A summary of the various test specimens is shown in Table 1, providing the sample codes to which the following work will refer, as well as the respective constituents for the single filler composites and the multiphase (MP) composites.

Table 1 Overview and nomenclature of prepared samples.

\begin{tabular}{|c|c|c|}
\hline Sample code & Sample nature & $\begin{array}{l}\text { Total amount } \\
\text { of additives }\end{array}$ \\
\hline $\mathrm{NE}$ & Neat epoxy & N/A \\
\hline G-POSS & $\begin{array}{c}\text { Epoxy + G-POSS } \\
1 \mathrm{wt} \%\end{array}$ & $1 \mathrm{wt} \%$ \\
\hline TG-POSS & $\begin{array}{c}\text { Epoxy + TG-POSS } \\
1 \mathrm{wt} \%\end{array}$ & $1 \mathrm{wt} \%$ \\
\hline cBN & Epoxy + c-BN 5wt\% & $5 \mathrm{wt} \%$ \\
\hline MP/G-POSS & $\begin{array}{c}\text { Epoxy + G-POSS } \\
1 w t \%+c-B N 5 w t \%\end{array}$ & $6 \mathrm{wt} \%$ \\
\hline MP/TG-POSS & $\begin{array}{l}\text { Epoxy+ TG-POSS } \\
\text { 1wt\% + c-BN 5wt\% }\end{array}$ & $6 \mathrm{wt} \%$ \\
\hline
\end{tabular}

The molecules of both, TG-POSS and G-POSS, feature distinct amounts of epoxy groups surrounding the silica-like core, which will consume curing agent during the reticulation process. As a result, distinctive stoichiometric ratios of epoxy, curing agent and the respective POSS were formulated for all POSScontaining composites, in order to balance the reactive epoxy groups of resin and POSS with the amine groups of the curing agent. The stoichiometric calculations were based on the respective epoxy equivalent weights of the constituents involved.

Considering the unique nature of the different fillers, distinct fabrication procedures were established to achieve a good dispersion of the additives in the epoxy matrix. The liquid additives, TG-POSS and G-POSS, were dispersed by means of a magnetic stir on a hot plate at a temperature of $40^{\circ} \mathrm{C}$. When only POSS was incorporated in the composites, following the aforementioned step, the curing agent was added to the liquid epoxy/POSS composite and mixed as well with a magnetic stir at $40^{\circ} \mathrm{C}$. Throughout the stirring procedures, vacuum was applied directly on the mixing vessel to degas the mixture before the liquid composites were cast. The method described has shown to give homogeneous epoxy/POSS composites, for the amounts of POSS used [12, 24].

When c-BN particles were involved, they were added either to the base epoxy, in the case of the cBN sample, or, in the cases of the two MP composites, the c-BN particles were added to the epoxy/POSS mixture, which was obtained according to the above mentioned description. Most importantly, before the curing agent was introduced, the liquid composites were sonicated, employing a high energy ultrasonic probe, to disperse the c-BN particles. After sonication, these liquid composites were thoroughly degassed, before the curing agent was added into the mixtures with a magnetic stir on a hotplate at $40^{\circ} \mathrm{C}$. Again, vacuum was applied directly on the mixing vessel during the multiple stirring procedures.

Subsequently, the liquid composites were cast in stainless steel molds and cured under vacuum at $100{ }^{\circ} \mathrm{C}$ for a period of six hours. The curing process followed a gradual cool down to room temperature. A release agent (QZ13) applied on the molds before the composites were cast, facilitated the demolding of the fabricated sample plaques.

\section{Experimental Methods}

\subsection{Microstructure analysis}

The test specimens containing submicrometric c-BN were immersed in liquid nitrogen to render them brittle and subsequently fractured in order to obtain crosssections for observations with a Scanning Electron Microscope (SEM). The two composites containing only POSS as an additive, viz. G-POSS and TG-POSS, were mounted in epoxy and subsequently polished with 
abrasive materials of gradually decreasing particle sizes down to $50 \mathrm{~nm}$ to obtain a very smooth surface for high magnification observations with the SEM.

For all composite samples, complete cross-sections with a width of approximately $2 \mathrm{~cm}$ and a height of $300 \mu \mathrm{m}$ were analyzed with an SU-70 high resolution SEM (Hitachi High Technologies America). Different magnification levels were chosen to assess the dispersion of the used filler in the epoxy matrix, depending on the respective filler sizes used for the composites. In the cases of POSS, magnifications of up to $\mathrm{x} 200 \mathrm{k}$ were employed, considering the very small size of the POSS molecules of around $2 \mathrm{~nm}$. When the submicrometric c-BN was involved in the composites, and hence, for the cBN and the two MP composites, magnifications of up to $\mathrm{x} 20 \mathrm{k}$ were sufficient to investigate the dispersion of the c-BN particles. Prior to the SEM observations, all samples were cleaned in an ultrasonic bath to remove debris from the fracturing process. Subsequently, the specimens were sputtercoated with a $2 \mathrm{~nm}$ Platinum layer to avoid charging due to the electron beam during the SEM observations.

\subsection{Dielectric spectroscopy}

The dielectric response was evaluated on samples $40 \mathrm{~mm}$ in diameter and with an average thickness of $300 \mu \mathrm{m}$ by the use of Broadband Dielectric Spectroscopy (BDS, Novocontrol Technologies GmbH) at an excitation voltage of $1 \mathrm{~V}$ and a temperature of $20^{\circ} \mathrm{C}$, for a frequency range of $0.1 \mathrm{~Hz}$ to $1 \mathrm{MHz}$. The complex permittivity $\varepsilon^{*}$ as given in (1) was obtained with the real permittivity $\varepsilon^{\prime}$, representing the real or storage part and $\varepsilon^{\prime \prime}$, the imaginary or dielectric loss part.

$$
\varepsilon(\omega)^{*}=\varepsilon^{\prime}(\omega)-i \varepsilon^{\prime \prime}(\omega)
$$

\subsection{Differential scanning calorimetry}

Glass transition temperatures $\mathrm{T}_{\mathrm{g}}$ were measured by Differential Scanning Calorimetry (DSC) with a heat flow calorimeter (Q20, TA Instruments). Each specimen was subjected to two thermal cycles with a heating rate of $10^{\circ} \mathrm{C} / \mathrm{min}$ from $20^{\circ} \mathrm{C}$ to $250{ }^{\circ} \mathrm{C}$, followed by a gradual cool down to $20^{\circ} \mathrm{C}$ also at $10^{\circ} \mathrm{C} / \mathrm{min}$. The $\mathrm{T}_{\mathrm{g}}$ were obtained from the second heating cycle to avoid any thermal history of the test specimens.

\subsection{AC breakdown strength}

The AC breakdown strength was retrieved based on the ASTM D 149 standard. Samples were immersed in transformer oil and positioned between two ball tip electrodes with a $4 \mathrm{~mm}$ diameter. The short-term test was used, where a $60 \mathrm{~Hz}$ voltage with a ramp rate of $2 \mathrm{kV} / \mathrm{s}$ was applied between the electrodes until breakdown occurred. Twelve breakdowns were carried out for each sample type, after which the ball tip electrodes were replaced to avoid pitting. The test specimens for this experiment had a thickness of $135 \mu \mathrm{m}$.

\subsection{Thermal conductivity}

The thermal conductivity was obtained with a DTC-25 guarded heat flow meter (TA Instruments) in accordance with the ASTM E 1530 standard. The circular samples, $50.8 \mathrm{~mm}$ ( 2 inches) in diameter and with an average thickness of $300 \mu \mathrm{m}$, were placed between the upper heating plate and the lower cooling plate of the instrument. Applying a pressure of 20 psi on the upper plate ensured intimate contact between the sample, the heating and the cooling plates. The thermal conductivity values were assessed for a mean sample temperature of $25^{\circ} \mathrm{C}$. The specimens remained inside the test chamber for two hours before the thermal conductivity values were obtained, to allow a thermal stabilization of the samples.

\section{Results and Discussion}

\subsection{Microstructure analysis}

The SEM micrographs revealed homogeneous bulk compositions for the G-POSS and TG-POSS samples (see Fig. 2a and Fig. 2b) without any particular trace of the POSS additives. Thus, both POSS additives seem to be dispersed into the polymeric matrix on a molecular level. In the case of the cBN sample (see Fig. 2c) a rather heterogeneous bulk material was observed, with agglomerated zones packing a high density of c-BN particles, while others were lacking c-BN. In comparison to this observation, it was found that in the cases of the two multiphase composites, MP/G-POSS and MP/TGPOSS (Fig. 2d and Fig. 2e, respectively), the incorporated c-BN particles were well dispersed throughout the bulk of the material. Thus, no clusters of c-BN particles were found for these composites.

It appears that the incorporation of the hybrid organic/inorganic POSS molecules in the organic epoxy network indeed resulted in some type of compatibilization with the inorganic c-BN. Due to the covalent bonds formed between the epoxy chains and the POSS molecules with its silica-like cores, the resulting epoxy/POSS network now features inorganic zones on a nanometric scale. This subsequently renders 


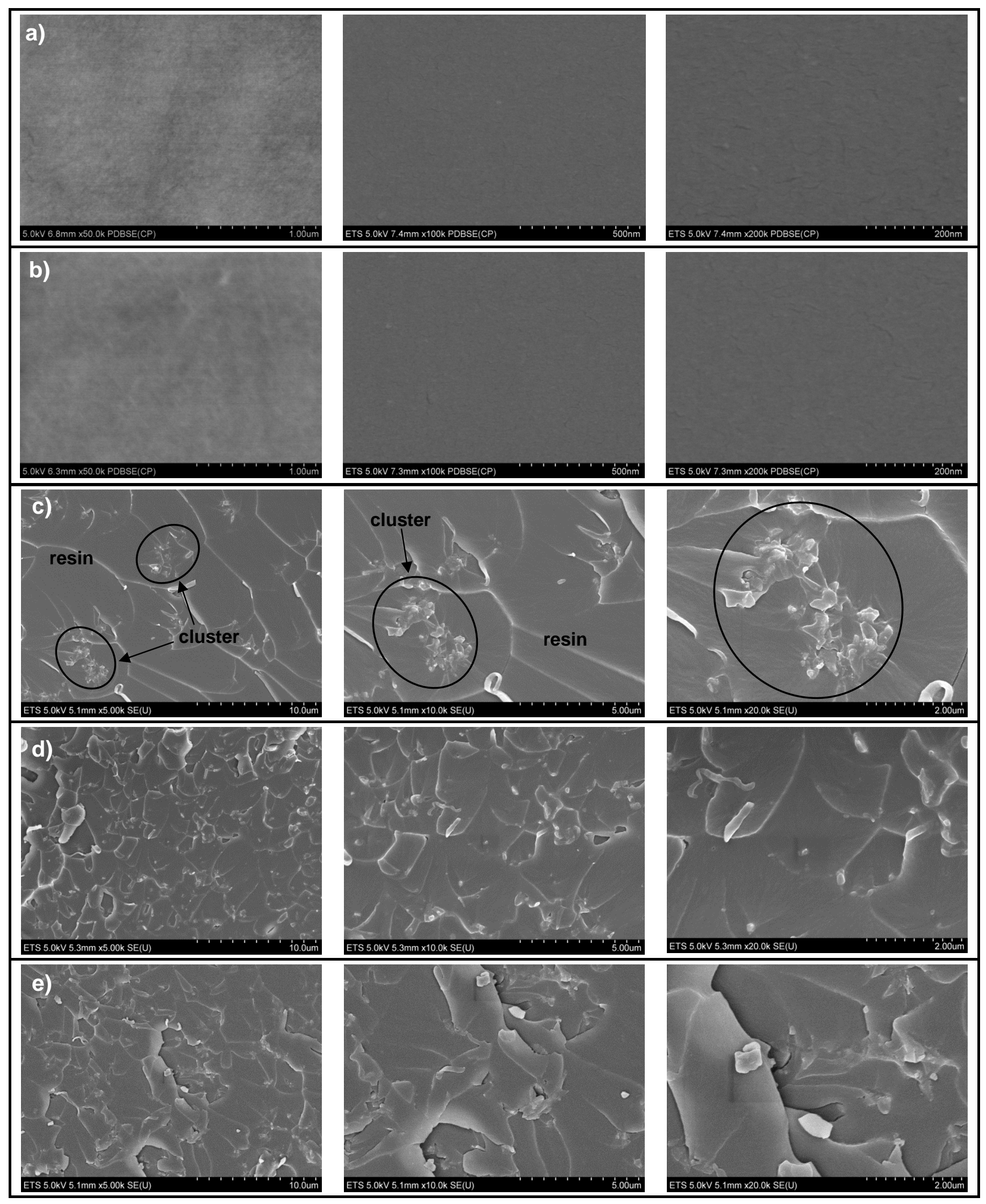

Fig. 2 Micrographs of the composites captured at different magnifications: (a) G-POSS, (b) TG-POSS, (c) cBN, (d) MP/G-POSS and (e) MP/TG-POSS. 
the per se organic epoxy into an intrinsically hybrid material with an organic backbone and inorganic silicalike regions (see Fig. 1). Thus, it is believed that POSS acts as a compatibilizer within the polymer backbone, which enables a homogeneous dispersion of the c-BN particles in the matrix. In recent studies [25, 26], POSS has been shown to perform as a functionalizing agent or compatibilizer by improving the interfacial interaction between filler particles and epoxies when the respective additives are surface treated with POSS. Consequently, homogeneous dispersions of the respective filler materials in the epoxy matrix were obtained in these studies.

\subsection{Dielectric spectroscopy}

The dielectric responses of the test specimens obtained show different results depending on the type of additive used, both in the real and in the imaginary parts of the complex permittivity. Compared with NE, the two single-phase POSS composites revealed slightly lower values of $\varepsilon^{\prime}$, which was more evident for the G-POSS sample (see Fig. 3a). This phenomenon has often been observed when low contents of nanometric additives are incorporated in a polymeric matrix [27, 28]. In contrary, for the cases involving c-BN, and hence, the cBN and the two multiphase composites, $\varepsilon^{\prime}$ was found to be higher than what was observed for NE, especially with decreasing frequencies. Since the values of the relative permittivity for c-BN increase from high frequency $\varepsilon_{\infty}=4.5$ towards the static $\varepsilon_{0}=7.1$ [19], it appears that the submicrometric c-BN particles dictate the dielectric permittivity for the $\mathrm{cBN}$ and MP composites.

Additionally, $\beta$-relaxation peaks were detected in $\varepsilon^{\prime \prime}$ for all samples in the vicinity of $100 \mathrm{kHz}$, as depicted in Fig. 3b. Those $\beta$-relaxation peaks are common for amorphous polymers and appear due to secondary relaxation processes caused by movements of local groups that are attached to the main polymer chain, namely hydroxyl ether groups, in the case of the epoxy system used. Similar to the observations made for $\varepsilon^{\prime}$, increased $\varepsilon^{\prime \prime}$ were also noted compared to neat epoxy, for the composites involving c-BN, and thus, the cBN and both MP samples.

In terms of both POSS composites though, the dielectric losses were found to be marginally lower for the frequency range from $1 \mathrm{kHz}$ to $0.1 \mathrm{~Hz}$, compared to the neat polymer. In the vicinity of the $\beta$-peak (around $100 \mathrm{kHz}$ ) however, the decrease in dielectric losses of both G-POSS and TG-POSS was more evident. It seems
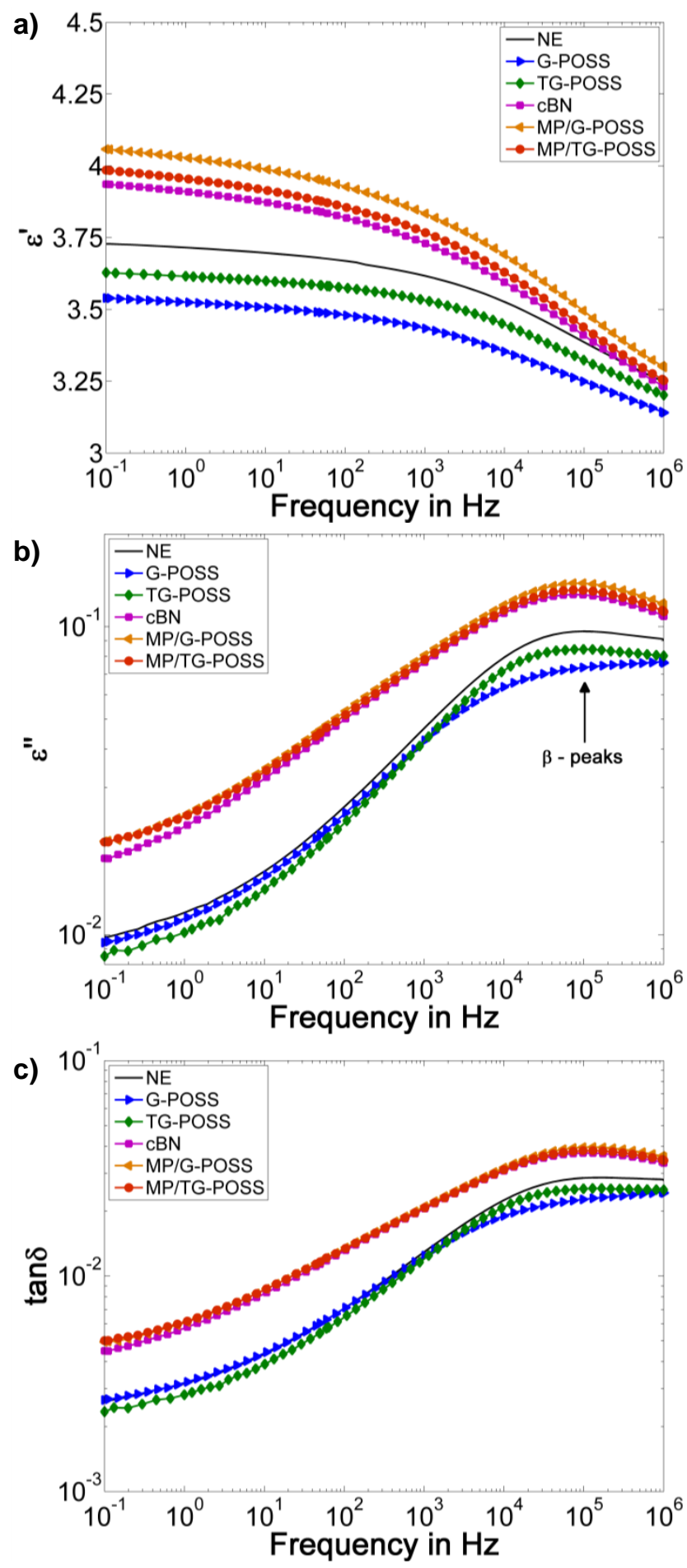

Fig. 3 Dielectric response of test specimens at $20^{\circ} \mathrm{C}$ and $1 \mathrm{~V}$; (a) real part of complex permittivity, (b) imaginary part of complex permittivity and (c) the resulting loss tangent.

that the incorporation of POSS in the matrix leads to an immobilization of the side groups contributing to the $\beta$ relaxation process, as described above. The immobilization can be explained by the formation of 
covalent bonds between POSS and epoxy molecules due to the reactive epoxy groups of POSS, which leads to a more rigid network in the POSS composites as compared to the neat polymer. This gets even more evident, when one compares the dielectric losses of the G-POSS composite, which are lower than those of TGPOSS in the vicinity of the $\beta$-peak. Since G-POSS has 8 to 12 reactive epoxy groups that can form covalent bonds with the epoxy matrix, compared to only three epoxy groups for the TG-POSS, it is evident that the resulting epoxy/POSS network is more rigid when GPOSS is added instead of TG-POSS, and hence, the lower dielectric losses for the G-POSS composite due to the $\beta$-relaxation process.

\subsection{Differential scanning calorimetry}

The DSC experiment revealed rather close values of $\mathrm{T}_{\mathrm{g}}$ which are summarized in Table 2 . The glass transition phenomenon is strongly interrelated with the chain mobility of the epoxy network, which itself will be dependent on factors such as the cross-linking degree, chain lengths, the flexibility of side-groups or chains, as well as free volume, as reported in this review [20]. Considering the close values of $\mathrm{T}_{\mathrm{g}}$ for $\mathrm{NE}$ and all of the composites, it can be concluded that the incorporation of either filler did not contribute to any significant changes to the mobility of the molecular chains of the epoxy or epoxy/POSS network during the glass transition process.

Table 2 Glass transition temperatures $T_{g}$ of test specimens.

\begin{tabular}{cc}
\hline Sample & $\mathrm{T}_{\mathrm{g}}\left({ }^{\circ} \mathrm{C}\right)$ \\
\hline NE & 92 \\
G-POSS & 91 \\
TG-POSS & 89 \\
cBN & 93 \\
MP/G-POSS & 93 \\
MP/TG-POSS & 92 \\
\hline
\end{tabular}

\subsection{AC breakdown strength}

The AC breakdown (BD) data obtained were processed with the two-parameter Weibull distribution according to the IEEE 930 standard, to evaluate the AC breakdown strengths of the test specimens:

$$
P\left(E_{B D}\right)=1-\exp \left[-\left(\frac{E_{B D}}{\alpha}\right)^{\beta}\right],
$$

where $P\left(E_{B D}\right)$ is the cumulative failure probability in \%, $E_{B D}$ is the experimental value of the dielectric breakdown strength in $\mathrm{kV} / \mathrm{mm}, \alpha$ is the scale parameter representing the breakdown strengths in $\mathrm{kV} / \mathrm{mm}$ at which $62.3 \%$ of the samples experienced electric BD, and the shape parameter $\beta$, which is an inverse measure for the scatter of the BD data, meaning the higher it is, the lower the variation of the data to the Weibull distribution. In accordance with the IEEE 930 standard, the cumulative failure probability for the experimental data of $E_{B D}$ can be approximated by:

$$
P(i, n)=\frac{i-0.44}{n+0.25}
$$

where $i$ denotes the result of the $i^{\text {th }}$ rank, when all values of $E_{B D, i}$ are sorted in ascending order, and $n$ represents the number of data points. In this work, $n=12$.

The Weibull plots of the BD experiment are shown in Fig. 4 and the respective Weibull parameters $\alpha$ and $\beta$ are listed in Table 3. Compared to the neat polymer NE, which had a BD strength of $211 \mathrm{kV} / \mathrm{mm}$, both POSS composites revealed higher AC BD strengths. TG-POSS had a slightly higher BD strength of $226 \mathrm{kV} / \mathrm{mm}$, whereas G-POSS revealed the highest BD strength, with $241 \mathrm{kV} / \mathrm{mm}$. In terms of the cBN composite and the two

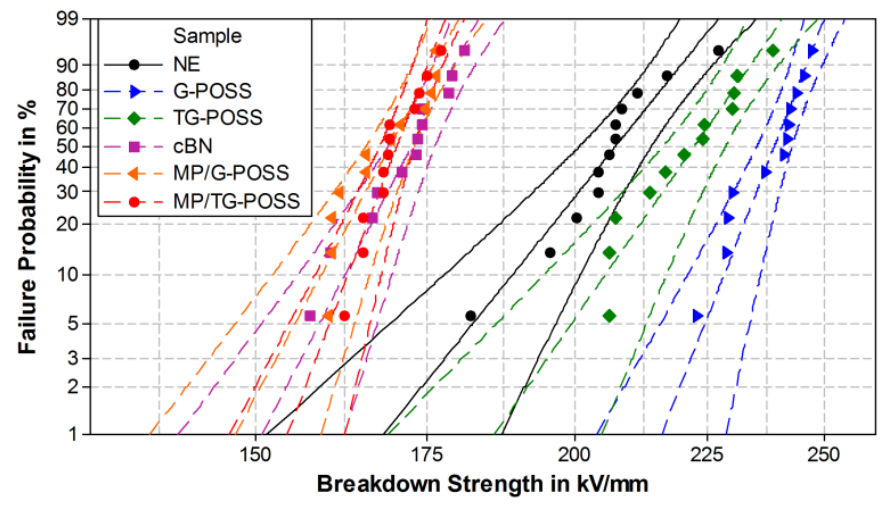

Fig. 4 Weibull plot of the breakdown data with $95 \%$ confidence intervals.

Table 3 Weibull parameters of the breakdown data.

\begin{tabular}{ccc}
\hline Sample & $\alpha(\mathrm{kV} / \mathrm{mm})$ & $\beta$ \\
\hline NE & 211 & 20.4 \\
G-POSS & 241 & 41.9 \\
TG-POSS & 226 & 23.7 \\
cBN & 174 & 31.5 \\
MP/G-POSS & 172 & 30.5 \\
MP/TG-POSS & 172 & 43.1 \\
\hline
\end{tabular}


multiphase (MP) composites though, significantly reduced $\mathrm{BD}$ strengths were recorded, compared to NE. With $174 \mathrm{kV} / \mathrm{mm}$ for the cBN composite and $172 \mathrm{kV} / \mathrm{mm}$ for both, the MP/G-POSS and MP/TGPOSS composites, it is evident that the submicrometric c-BN particles dominate the breakdown behavior of the multiphase composites. The decrease in BD strength in the presence of the c-BN particles can be attributed to local electrical field enhancements in the proximity of cBN caused by the irregular shape of the filler particles which feature pronounced edges, and also due to the mismatch of the dielectric permittivity, compared to epoxy [6, 29, 30].

It has been argued in the past, i.e. [31, 32], that some of the benefits of incorporation of POSS into a polymeric matrix are due to the ability of POSS to structure a polymeric network on a nanometric level, which occurs by the formation of covalent bonds between the reactive POSS types used and the epoxy, and thus leads to a more homogeneous molecular structure of the epoxy/POSS network. This fact, plus a local reinforcement of the polymer chains with inorganic silica, should give rise to the enhanced BD strengths of the POSS composites.

\subsection{Thermal conductivity}

The obtained thermal conductivities of the test specimens are summarized in Table 4. Our experiments showed a thermal conductivity of $0.143 \mathrm{~W} / \mathrm{m} \cdot \mathrm{K}$ for NE, which is in accordance with previously published findings for this epoxy resin [12, 21]. Based on these values, all composite materials were found to have increased thermal conductivities. The TG-POSS composite has seen only a slightly increased thermal conductivity of $0.156 \mathrm{~W} / \mathrm{m} \cdot \mathrm{K}$, whereas for the G-POSS specimen, a $20 \%$ enhancement in thermal conductivity

Table 4 Evaluation of thermal conductivity measurements.

\begin{tabular}{ccc}
\hline Sample & $\begin{array}{c}\text { Thermal conductivity } \\
(\mathrm{W} /(\mathrm{m} \cdot \mathrm{K}))\end{array}$ & $\begin{array}{c}\text { Relative } \\
\text { values }\end{array}$ \\
\hline $\mathrm{NE}$ & 0.143 & $100 \%$ \\
G-POSS & 0.172 & $120 \%$ \\
TG-POSS & 0.156 & $109 \%$ \\
cBN & 0.180 & $126 \%$ \\
MP/G-POSS & 0.178 & $125 \%$ \\
MP/TG-POSS & 0.177 & $124 \%$ \\
\hline
\end{tabular}

to $0.172 \mathrm{~W} / \mathrm{m} \cdot \mathrm{K}$ was achieved. The cBN composite, however, revealed the most important improvement in thermal conductivity, with a value of $0.180 \mathrm{~W} / \mathrm{m} \cdot \mathrm{K}$, representing an increase of more than $25 \%$ compared to NE. Considering the rather similar values obtained for the multiphase composites, viz. $0.178 \mathrm{~W} / \mathrm{m} \cdot \mathrm{K}$ for MP/GPOSS and $0.177 \mathrm{~W} / \mathrm{m} \cdot \mathrm{K}$ for MP/TG-POSS, it is evident that no synergetic effect in terms of a further improved thermal conductivity could be attained by incorporation of both POSS and c-BN together in a multiphase composite.

It is well established that the thermal conduction in dielectric solids is based on phonons (lattice vibrations) $[5,33]$. However, in amorphous epoxy resins the dominating process is phonon scattering, which causes a diminishment of thermal conductivity within a material. Phonon scattering can occur at matrix-filler or fillerfiller interfaces, as well as at the interfaces of the amorphous structure in epoxy resins itself [5]. The incorporation of low amounts of POSS in epoxy resins is believed to bring about a re-structuration of the resulting composites' molecular structure, caused by covalent bonds between epoxy and POSS. Furthermore, the reticulation between filler and matrix is considered to diminish phonon scattering in the TG-POSS and especially in the G-POSS composite, and thus leads to an enhancement of the POSS composites' thermal conductivities compared to the neat polymer.

The superior performance of the G-POSS composite in comparison with TG-POSS can accordingly be attributed to the higher degree of reactivity of G-POSS, with its 8 to 12 epoxy groups, instead of only 3 epoxy groups in the case of the TG-POSS. This allows the GPOSS to better integrate in the matrix, creating more continuous pathways for the heat conduction by interconnecting epoxy molecules with one another. In the multiphase composites, however, the c-BN particles with their comparatively large size of $500 \mathrm{~nm}$ apparently obstruct the described effect of POSS on the epoxy network. Thus, no beneficial synergetic effect of POSS and c-BN on the thermal conductivity was seen for the multiphase composites. In conclusion, the c-BN particles also dominate the heat conduction properties of both, the MP/G-POSS and MP/TG-POSS composite.

\section{Concluding Remarks}

Both POSS samples, but especially the G-POSS composite, have shown notably enhanced properties in terms of BD strength and dielectric losses, as well as 
increased thermal conductivities compared to the neat polymer. However, in the presence of the submicrometric c-BN particles in the two multiphase composites, the re-structuration of the polymeric matrix by incorporation of POSS seems to be compromised by the comparatively large c-BN particles, with an average size of $500 \mathrm{~nm}$. Thus, no additional benefit was found in terms of thermal conductivity or BD strength when both POSS and c-BN were incorporated together in a multiphase (MP) sample.

On the contrary, when comparing G-POSS with the cBN and the MP samples, besides the marginally higher thermal conductivity of cBN and both MP composites, it is evident that the G-POSS specimen features the overall best performance of a dielectric material for high voltage insulation, with lower dielectric losses, higher BD strength, as well as increased thermal conductivity, compared to the base epoxy.

It is however most intriguing that even the clearly improved dispersion of the introduced c-BN particles in the MP composites, as presented in Fig. 2 did not contribute to any kind of enhancement in either electric or thermal performance of the respective composites. Consequently, it is believed that the choice of the submicrometric c-BN particles used was inadequate to benefit from the structuring abilities of POSS. Considering that the c-BN particles used were comparatively large, with an average size of $500 \mathrm{~nm}$ compared to approximately $2 \mathrm{~nm}$ for the POSS molecules, it might be of interest to conduct a similar study involving a nanometric sized filler, with sizes closer to that of the POSS molecules used, in order to avoid the perturbation of the POSS' structuring effect, and thus, enable a synergetic effect of the two fillers.

Although no complex chemical surface treatment of the c-BN particles involved was applied in our study, yet a homogeneous dispersion of the inorganic c-BN particles was seen for the multiphase composites (see Fig. 2d and Fig. 2e), this effect of POSS on the compatibility between polymer and filler should be regarded as a major point of interest in nano-dielectrics or nano-composites in general. In fact, the dispersion of nanometric inorganic filler particles within polymers is still a very current problematic [13, 23, 28, 34]. The possibility of using POSS as a compatibilizer in industrial manufacturing processes instead of more complex chemical procedures, which might come with certain health or environmental risks, and hence, the approach of formulating epoxy composites combining reactive POSS and other additives to improve their dispersion within the epoxy matrix, could significantly contribute to the advancement of the implementation of epoxy-based nano-composites on an industrial level.

\section{Acknowledgment}

Hydro-Québec and the Natural Sciences and Engineering Research Council of Canada (NSERC) are gratefully thanked for their financial support.

\section{References}

[1] L. A. Dissado and J. C. Fothergill, Electrical degradation and breakdown in polymers. London: P. Peregrinus, 1992.

[2] I. A. Tsekmes, R. Kochetov, P. H. F. Morshuis, and J. J. Smit, "Modeling the thermal conductivity of polymeric composites based on experimental observations," IEEE Transactions on Dielectrics and Electrical Insulation, vol. 21(2), pp. 412-423, 2014.

[3] T. Tanaka, Z. Wang, T. Iizuka, M. Kozako, and Y. Ohki, "High thermal conductivity epoxy/BN composites with sufficient dielectric breakdown strength," in Power and Energy Systems (ICPS), 2011 International Conference on, 2011, pp. 1-4.

[4] R. Kochetov, T. Andritsch, U. Lafont, P. H. F. Morshuis, and J. J. Smit, "Thermal conductivity of nano-filled epoxy systems," in IEEE Conference on Electrical Insulation and Dielectric Phenomena (CEIDP), 2009.

[5] Y. Takezawa, M. Akatsuka, and C. Farren, "High thermal conductive epoxy resins with controlled high order structure," in Properties and Applications of Dielectric Materials, 2003. Proceedings of the 7th International Conference on, 2003, pp. 1146-1149 vol.3.

[6] T. Heid, M. Fréchette, and E. David, "Epoxy/BN Micro- and SubmicroComposites: Dielectric and Thermal Properties of Enhanced Materials for High Voltage Insulation Systems," IEEE Transactions on Dielectrics and Electrical Insulation, vol. 22(2), pp. 1176-1185, 2015.

[7] J. Abenojar, M. A. Martínez, M. Pantoja, F. Velasco, and J. C. Del Real, "Epoxy Composite Reinforced with Nano and Micro SiC Particles: Curing Kinetics and Mechanical Properties," Journal of Adhesion, vol. 31(2), pp. 418-434, 2012.

[8] A. Baker, S. Dutton, and D. Kelly, Composite Materials for Aircraft Structures (2nd Edition): American Institute of Aeronautics and Astronautics, 2014.

[9] J. K. Nelson and J. C. Fothergill, "Internal charge behaviour of nanocomposites," Nanotechnology, vol. 15(5), p. 586, 2004.

[10] E. Tuncer, I. Sauers, D. R. James, A. R. Ellis, M. P. Paranthaman, T. Aytuğ, et al., "Electrical properties of epoxy resin based nanocomposites," Nanotechnology, vol. 18(2), p. 025703, 2007.

[11] J. K. Nelson and Y. Hu, "Nanocomposite dielectrics-properties and implications," Journal of Physics D: Applied Physics, vol. 38(2), p. 213, 2005.

[12] T. Heid, M. Fréchette, and E. David, "Nanostructured Epoxy/POSS composites: Enhanced Materials for High Voltage Insulation Applications," IEEE Transactions on Dielectrics and Electrical Insulation, vol. Vol. 22(3), pp. 1594-1604, 2015.

[13] J. K. Nelson, Dielectric Polymer Nanocomposites: Springer Science+Business Media, 2010.

[14] X. Huang, Y. Li, F. Liu, P. Jiang, T. Iizuka, K. Tatsumi, et al., "Electrical properties of epoxy/POSS composites with homogeneous nanostructure," Dielectrics and Electrical Insulation, IEEE Transactions on, vol. 21(4), pp. 1516-1528, 2014.

[15] T. Heid, M. Fréchette, and E. David, "Nanostructured epoxy/POSS composites: High performance dielectrics with improved breakdown strength and corona resistance," in Electrical Insulation and Dielectric Phenomena (CEIDP), 2014 IEEE Conference on, 2014, pp. 659-662.

[16] T. Heid, M. Fréchette, and E. David, "Nanostructured epoxy/POSS composites: High performance dielectrics with improved corona resistance and thermal conductivity," in Electrical Insulation Conference (EIC), 2014, 2014, pp. 316-319.

[17] Z. Wang, T. lizuka, M. Kozako, Y. Ohki, and T. Tanaka, "Development of epoxy/BN composites with high thermal conductivity and sufficient dielectric breakdown strength part I - sample preparations and thermal 
conductivity," IEEE Transactions on Dielectrics and Electrical Insulation, vol. 18(6), pp. 1963-1972, 2011.

[18] R. Kochetov, T. Andritsch, P. H. F. Morshuis, and J. J. Smit, "Effect of filler size on complex permittivity and thermal conductivity of epoxybased composites filled with BN particles," in IEEE Conference on Electrical Insulation and Dielectric Phenomena (CEIDP), 2010.

[19] Y. Kumashiro, Electric refractory materials. New York: Marcel Dekker, 2000.

[20] F. Klocke, Manufacturing Processes 1 Cutting. Berlin, Heidelberg: Springer-Verlag Berlin Heidelberg, 2011.

[21] T. Heid, M. Fréchette, and E. David, "Dielectric and thermal properties of submicrometric epoxy/c-BN composites," in IEEE Conference on Electrical Insulation and Dielectric Phenomena (CEIDP), 2014, pp. 719-722.

[22] M. Dongling, A. H. Treese, W. S. Richard, C. Anna, M. Eva, Ö. Carina, et al., "Influence of nanoparticle surface modification on the electrical behaviour of polyethylene nanocomposites," Nanotechnology, vol. 16(6), pp. 724-731, 2005.

[23] T. Tanaka, T. lizuka, Y. Ohki, X. Huang, and P. Jiang, "Dielectric properties and thermal conductivity of epoxy/AIN composites with several kinds of filler-matrix interfaces," in IEEE International Conference on Solid Dielectrics (ICSD), 2013, pp. 377-380.

[24] T. Heid, M. Fréchette, and E. David, "Dielectric and Thermal Properties of Nanostructured Epoxy Composites Involving Reactive POSS and cBN," in IEEE Electrical Insulation Conference (EIC 2015), Seattle, 2015.

[25] X. Huang, T. Iizuka, P. Jiang, Y. Ohki, and T. Tanaka, "Role of Interface on the Thermal Conductivity of Highly Filled Dielectric Epoxy/AlN Composites," The Journal of Physical Chemistry C, vol. 116(25), pp. 13629-13639, 2012/06/28 2012.

[26] X. Huang, C. Zhi, P. Jiang, D. Golberg, Y. Bando, and T. Tanaka, "Polyhedral Oligosilsesquioxane-Modified Boron Nitride Nanotube Based Epoxy Nanocomposites: An Ideal Dielectric Material with High Thermal Conductivity," Advanced Functional Materials, vol. 23(14), pp. 1824-1831, 2013.

[27] A. Krivda, T. Tanaka, M. Fréchette, J. Castellon, D. Fabiani, G. C. Montanari, et al., "Characterization of epoxy microcomposite and nanocomposite materials for power engineering applications," IEEE Electrical Insulation Magazine, vol. 28(2), pp. 38-51, 2012.

[28] R. Kochetov, T. Andritsch, P. H. F. Morshuis, and J. J. Smit, "Anomalous behaviour of the dielectric spectroscopy response of nanocomposites," IEEE Transactions on Dielectrics and Electrical Insulation, vol. 19(1), pp. 107-117, 2012.

[29] R. Lovell, "The Effect of Specimen Size on the Electric Breakdown of Unfilled and Filled Epoxy Polymers," Electrical Insulation, IEEE Transactions on, vol. EI-11(4), pp. 110-114, 1976.

[30] Q. Wang, Z. Li, J. Wu, and Y. Yin, "The thermal conductivity and electrical strength of epoxy resin with different filler content of micro and nano alumina," in Condition Monitoring and Diagnosis (CMD), 2012 International Conference on, 2012, pp. 1110-1113.

[31] A. Strachota, P. Whelan, J. Křŕž, J. Brus, M. Urbanová, M. Šlouf, et al., "Formation of nanostructured epoxy networks containing polyhedral oligomeric silsesquioxane (POSS) blocks," Polymer, vol. 48(11), pp. 3041-3058, 2007.

[32] J. Chen, "Nanostructuration of epoxy networks by using polyhedral oligomeric silsesquioxanes POSS and its copolymers," PhD, École Doctorale des Matériaux I. M. P. Ingénierie des Matériaux Polymères, Institut national des sciences appliquées, Lyon, 2012.

[33] W. Cui, F. Du, J. Zhao, W. Zhang, Y. Yang, X. Xie, et al., "Improving thermal conductivity while retaining high electrical resistivity of epoxy composites by incorporating silica-coated multi-walled carbon nanotubes," Carbon, vol. 49(2), pp. 495-500, 2011.
[34] R. Y. Hong and Q. Chen, "Dispersion of Inorganic Nanoparticles in Polymer Matrices: Challenges and Solutions," in Organic-Inorganic Hybrid Nanomaterials. vol. 267, S. Kalia and Y. Haldorai, Eds., ed: Springer International Publishing, 2015, pp. 1-38.

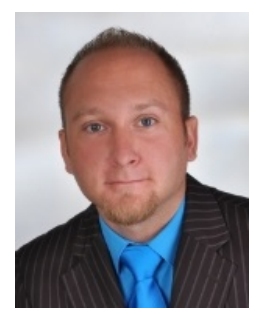

Thomas Heid (S’10) studied electrical engineering at Graz University of Technology (Austria) where he graduated with an M.Sc. (2011) degree, specializing in high voltage engineering. He was a project assistant at the Institute of High Voltage Engineering and System Management and the Test Institute of High Voltage Engineering (Graz, Austria) until 2012, where he has been involved in numerical calculations for high voltage cable systems, life cycle analysis for stator bars, as well as high voltage testing of electrical equipment. Currently he is a Ph.D. candidate with the Hydro-Québec Research Institute (IREQ) in cooperation with the École de Technologie Supérieure (ETS). His main research interest is the development of nanodielectrics for application in high voltage insulation systems.

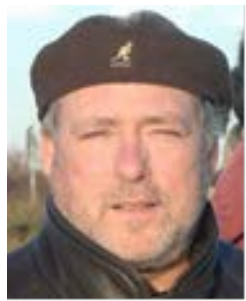

Dr. Michel Fréchette has a graduate degree in physics. He joined HydroQuébec's Research Institute (IREQ) in the $20^{\text {th }}$ century. Since then, he has been involved in applied basic research in various fields related to electrical insulation and insulating materials. His major contributions involve discharge physics, dielectric phenomena and nanodielectrics.

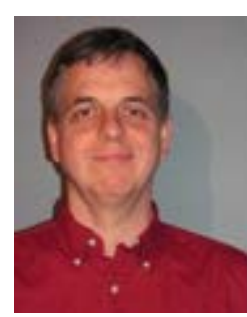

Eric David (M'03, SM'07) was born in Montreal in 1965. He received the M.Sc.A and Ph.D. degrees in Engineering Physics from the École Polytechnique de Montréal in 1989 and 1996, respectively. He joined the Hydro-Québec Research Institute (IREQ) in 1998, and from 2001 to 2002, where he was active in the field of dielectric materials used for underground cables and rotating machines. He is now a Professor at the Department of Mechanical Engineering in the École de Technologie Supérieure (ETS) in Montreal. His research interests include dielectric and nanodielectric materials, rotating machinery and underground cable insulation. He is an Associate Editor of the IEEE Transactions on Dielectrics and Electrical Insulation. 